\title{
FAMILIA Y ORIENTACIÓN LABORAL EN LA CONFORMACIÓN DEL OBJETIVO PROFESIONAL DE JÓVENES UNIVERSITARIOS EN BÚSQUEDA DE EMPLEO
}

\author{
Family and Labor Orientation in the Conformation \\ of the Professional Objective of Young University Students \\ in Search of Employment
}

\author{
Antonio Martínez López \\ Doctor en Sociología por la Universidad de Granada \\ Departamento de Sociología de la Universidad de Granada
}

Recibido: $16-12-2017$

DOI: $10.1387 /$ lan-harremanak.20086

Aceptado: 10-01-2018

\section{ABSTRACT}

Las politicas activas de empleo se han convertido en una herramienta unánimemente calificada como fundamental por los politicos y la administración para intervenir en una situación de altas tasas de paro estructural como la que estamos atravesando en la actualidad. Aunque no muy numerosos, no son pocos los trabajos de tipo macro sociológico y económico que hay sobre las politicas activas de empleo, especialmente sobre la formación para los parados. Sin embargo muy pocos que aborden una micro sociología de la relación que se da entre los jóvenes universitarios y el uso de la orientación laboral en sus estrategias de búsqueda de empleo, que es lo que novedosamente propone este articulo.

En el trabajo se llega a algunas conclusiones interesantes y en algunos aspectos novedosos, en relación con la forma en que se elaboran las estrategias educativas y labora- 
les de los egresados universitarios en relación con su ubicación social y su consecución del logro ocupacional. Más allá de la clase social de la familia, se aporta una categorización de configuraciones familiares en relación con ese proceso de transición, que demuestran la centralidad de los capitales escolares y sociales de los padres para la consecución de los objetivos de los jóvenes universitarios, y el papel que las PAEs juegan en ese contexto, que siendo muy residual en términos de empleo y mercado laboral, es justo admitirles funciones latentes de poco calado pero que pueden apreciarse de manera clara al trazar las trayectorias de los jóvenes universitarios.

Palabras clave: Jóvenes universitarios, orientación laboral, búsqueda de empleo, familia, formación.

Active employment policies have become a tool unanimously qualified as fundamental by politicians and the administration to intervene in a situation of high rates of structural unemployment like the one we are going through today. Although not very numerous, there are many macro-sociological and economic works on active employment policies, especially on training for the unemployed. However, very few that address a micro sociology of the relationship that exists between university students and the use of career guidance in their job search strategies, which is what this article proposes.

In the paper, we come to some interesting conclusions and in some novel aspects, in relation to the way in which the educational and work strategies of university graduates are elaborated in relation to their social location and their attainment of occupational achievement. Beyond the social class of the family, a categorization of family configurations is provided in relation to this process of transition, which demonstrate the centrality of the school and social capital of parents to achieve the objectives of university students, and the role that the PAEs play in this context, that being very residual in terms of employment and the labor market, it is fair to admit latent functions of little depth but that can be clearly appreciated when tracing the trajectories of university students.

Keywords: University students, career guidance, job search, family, training. 


\section{SUMARIO}

1. Introducción. 2. Teoría y método. 3. Configuraciones familiares y formación de los objetivos profesionales en los buscadores de empleo. 3.1. Hipótesis sobre estructuración de configuraciones laborales y su relación con la estrategia de construcción y persecución del logro educativo. 3.1.1. Dimensiones centrales sobre las que se articulan las configuraciones familiares. 4. Configuraciones familiares, orientación laboral y trayectorias de búsqueda de empleo. 5. Conclusión. 6. Bibliografía.

\section{Introducción}

La crisis en la que están inmersas las economías de la Euro Zona desde los 2007-8 tienen como consecuencia un hecho mayor e insoslayable: el crecimiento del paro. Pero si bien esta crisis tiene su origen en el sistema financiero, marcado especialmente por el contagio a Europa de la caída de Lehmann Brothers en los EEUU, el aspecto local y nacional de esta destrucción global de valor y de este proceso de reordenación y de reestructuración internacional del capital es su impacto sobre el tejido productivo de nuestro país (significativamente, la debacle del inflaccionado sector de la construcción), y en última instancia sobre su población activa, sobre su población ocupada y sobre su población parada.

Quizás el fenómeno que representa con mayor contundencia la descomposición de un modelo de relación de las fuerzas productivas sin que se vea por el momento hacia donde apunta la salida de esta transición del modo de producción, sea el del fuerte e intenso aumento de las tasas de paro de jóvenes con menos de treinta ańos dentro del paro por grupos de edad, aumento que ha sido el más rápido y el más voluminoso en España en el marco de la Europa de los quince, en los dos primeros años de la crisis 2008-2010, Erhel (2010: 10). Tendencia que no se ha alterado hasta nuestros días, instalando a España a la cabeza en paro Juvenil de la Europa de los quince.

Por lo tanto, si hay una tendencia histórica a la desregulación del trabajo como trasfondo de la crisis internacional, lo cierto es que hay que entender el paro y la búsqueda de empleo en el marco de configuraciones sociales de empleo o regímenes de empleo, que como muy bien nos explican Prieto y Miguelez (2009) son las resultantes históricas y nacionales de una determinada composición entre la naturaleza y estructura de puestos de trabajo en el mercado, el modelo jurídico contractual de las relaciones laborales y la protección del desempleo asociada al modelo de bienestar social de cada país. 
¿Y cuál es la política social y de empleo que pone en marcha la clase política española para hacer frente a una crisis laboral real de las magnitudes que estamos describiendo? Pues una política de empleo basada fundamentalmente en la inercia de reclamar la aplicación de Políticas Activas de Empleo (que en lo sucesivo llamaremos PAEs). Estas medidas no representan más que el enfoque del neoliberalismo económico en materia de políticas públicas, que han sido impulsadas por la Estrategia Europea del Empleo a base de argumentaciones vagas y contradictorias con respecto al mantenimiento de la cohesión social que paradójicamente predican sus postulados genéticos (Crespo Suárez y Serrano Pascual, 2007), y de cuya eficacia en la mejora del ajuste entre oferta y demanda de trabajo no han sido capaces de dar cuenta más que en discursos oficiales, como el de la Estrategia Europea del Empleo (Martínez, 2011), eso sí con una constante renovación de su impulso ideológico y de un optimismo antropológico en sus virtualidades muy poco relacionado con el espíritu empírico que debería acompañar los enfoques en materia de paro y de cohesión social.

De hecho, algunos estudios sobre el impacto de la crisis sobre las políticas sociales y de empleo de los países europeos, muestran que si bien una tendencia general de Europa entre los años 2008 y 2010 ha sido la de primar el polo de la flexibilidad frente al de la seguridad, sin embargo los países nórdicos y continentales han hecho esfuerzos contradictorios en la protección del desempleo y en la aplicación de políticas keynesianas en países nórdicos y continentales, mientras que nada de ello ha sucedido en los países del sur, y especialmente nada en el sentido de reforzar la seguridad del empleo ha sucedido en España (Erhel, 2010). España confía en el modelo de sociedad familista para amortiguar los dańos sociales colaterales de la desorientación del capitalismo financiero que actualmente rige y dirige la globalización.

Esto nos lleva directamente a pensar en el importante papel que podría estar jugando la familia como amortiguador de la crisis laboral que se cierne especialmente sobre las poblaciones juveniles, dentro de la lógica que ya describen Sarasa y Moreno (1995) para países donde como el nuestro, los vaivenes de la creación de riqueza a nivel de mercado de trabajo son escasamente rectificados por las estructuras del Estado del Bienestar. Venimos de plantearlo justamente.

Y efectivamente el objetivo central de este artículo se centrará en ver sobre qué estructura de apoyo central reposa el proceso de búsqueda de empleo de los jóvenes egresados del mercado laboral, que se demuestra claramente conformado por el universo familiar (las configuraciones familiares que explicaremos con todo detalle) y qué papel puede asignarse a medidas públicas institucionalizadas como la orientación laboral, fundamentalmente, y los cursos de Formación profesional ocupacional que la complementan.

No son pocos los estudios españoles que parten del concepto de familia para ver la relación entre ésta y el proceso educativo y formativo de los hijos, que está 
en la base de la posterior división del trabajo en la sociedad de mercado. En este sentido podemos hablar del trabajo de Martínez (2007), donde analiza la relación entre clase social de la familia y desigualdades educativas en la Comunidad Canaria, o el trabajo de Martín y otros (2000), que focaliza su estudio en las familias de clase obrera y su relación con el logro educativo. Resulta conveniente destacar este tipo de estudios por partir habitualmente de la definición de un concepto de clase social, normalmente en base a indicadores sociolaborales, que es previo al trabajo de análisis del material empírico de los estudios.

En este sentido hay que recalcar el proceder inverso que operamos en este trabajo, y que quizás constituya una cierta novedad en campo de la Sociología de las transiciones educación-empleo. Aquí partimos del concepto de configuración familiar de Bernard Lahire, para descubrir y proponer un esquema de configuraciones en que se estructuraría la familia en su apoyo escolar y laboral a los hijos, a partir de las evidencias empíricas cualitativas de diez trayectorias de búsqueda de empleo.

Constatada empíricamente la importancia crucial de la familia en la transición estudio-trabajo propia de una sociedad de conocimiento como la actual el objetivo del trabajo se completa ubicando y explicando el papel que jugaría la orientación laboral dentro de las estrategias desplegadas por jóvenes con formación a la hora de fijar su objetivo profesional y situarse en el mercado laboral. En este trabajo quedan refutadas, sin necesidad de recurrir a una confrontación directa, las teorías de Gary Becker que, alineadas con el pensamiento económico neoclásico y con el neoliberalismo imperante en materia de políticas de empleo, consideran la formación y las políticas de la oferta como fundamentales para explicar el acceso al empleo. O más recientemente también el importantísimo papel en términos de mediadores laborales que se les otorga a estas medidas, por parte de (Pennel, 2013). Nosotros mostramos aquí que la orientación y los cursos de FPO tienen un papel muy secundario en la consecución de logros ocupacionales, casi despreciable, siendo la familia la variable que interviene de forma decisiva en relación con las transiciones juveniles. Es la configuración familiar la que sirve a construir el capital social de los jóvenes buscadores de empleo, además de intervenir en la propia articulación del logro ocupacional deseado.

\section{Teoría y método}

Dos autores resultan centrales para el estudio de la formación del objetivo profesional y su relación con la orientación laboral, tal y como lo desarrollamos aquí: se trata del eminente sociólogo francés Pierre Bourdieu y del no menos destacada sociólogo americano Erving Goffman. Dos obras centrales de Goffman aparecen en el sustrato epistemológico de este trabajo. Entenderemos el concepto de Trayectoria estudio-empelo, en el sentido que le da Goffman a 
su concepto de carrera moral, expuesto por el autor en su celebrada obra «Internados: ensayos sobre la situación social de los enfermos mentales». La carrera moral del buscador de empleo consiste en un proceso en el que se atraviesa por una serie de fases que implican la confianza o la distancia frente a los distintos instrumentos y apoyos que las personas van usando en su proceso de búsqueda.

De Pierre Bourdieu resulta central para el esqueleto argumental de este artículo el uso de sus tres conceptos centrales: capital, campo y hábitus. Sobre el propio concepto de hábitus, el sociólogo francés, que fuera discípulo de Bourdieu, Bernard Lahire, construye el concepto de "Configuración Familiar». Este concepto entronca con el pensamiento sociológico relacional de Pierre Bourdieu, donde la explicación sociológica no debe de dar por sentado el significado de las variables relacionándolas a modo de causa efecto a posteriori de una supuesta esencia, sino que justamente debe de explicar cómo se conforman las propias variables, es decir, lo que cada una significa sociológicamente en relación con la otra, y más allá de la constancia significativa que el convencionalismo nominal les presuponen en el análisis.

El concepto de Configuración Familiar de Bernard Lahire, viene muy bien expuesto en su libro: «Tableaux de Familles», concepto que pasaremos a explicar más en profundidad a continuación, de modo que resulta clave para caracterizar las 10 trayectorias de búsqueda de empleo analizadas.

Se han efectuado diez entrevistas en profundidad a egresados universitarios, situados en distintos momentos de su carrera moral en relación con el empleo (diacronía de las fases, para comprenderlas dentro de su relación familia-orientación), captados en una misma fase sincrónica de estas trayectorias: el paso por los servicios de orientación laboral universitaria en búsqueda de inserciones laborales. El carácter diacrónico del estudio de las carreras morales es necesario para comprender la estrategia de búsqueda y el papel que juegan actores y PAEs en su desarrollo. Esto se ha resuelto por el seguimiento de tres años a partir de la entrevista de muy larga duración efectuada a cada uno de los diez postulantes al trabajo entrevistados.

El marco geopolítico donde se enmarca el análisis es el de una ciudad y provincia Andaluza como es Granada, históricamente conocida por su escasa o nula presencia de trabajos de alto valor ańadido, con un tejido empresarial que opera fundamentalmente en el sector servicios, hostelería, restauración, y con una importante presencia de economía agraria de subsistencia en muchos casos. Por lo tanto con muy poca capacidad de absorción de empleo cualificado en su seno, lo que coloca a los jóvenes urbanizados formados en la Universidad en la necesidad de optar por la movilidad residencial a la que se resisten, a pesar de las llamadas constantes a dicha movilidad por los orientadores laborales. Por lo tanto, partimos de que el escenario donde han moverse los jóvenes universitarios hoy es el mismo, o si cabe peor, que el que se daba en el momento del análisis, hace 
diez años, como lo corroboran los propios datos del Observatorio de las Ocupaciones del SEPE (2017), donde se corrobora un estancamiento de la creación de empleo frente al crecimiento del PIB nacional y local, propio de la estructura del mercado de trabajo que definimos con anterioridad. Sólo un dato para simbolizar el marco geopolítico y laboral de estudio, la tasa de paro de la Provincia de Granada en el tercer trimestre de 2017, asciende a 26.95 de la población activa, mientras que la tasa nacional en el mismo momento es de 16.38 (datos extraídos del INE en noviembre de 2017), y ni que decir tiene que Granada está entre las tasas más altas de desempleo juvenil del territorio nacional.

\section{Configuraciones familiares y formación de los objetivos profesionales en los buscadores de empleo}

Bernard Lahire (1995: 18) considera dos espacios que pueden entenderse como generadores diferenciados de conjuntos de interdependencias sobre las que se estructuran una pluralidad de configuraciones, que al final conducen a diferentes estilos o modelos de aprendizaje. Estas dos esferas o espacios son la familia y el sistema educativo.

En nuestro intento por comprender cómo y dónde intervienen las PAEs en las tareas y estrategias que las personas llevan a cabo para ubicarse en el escenario laboral, no podíamos, al igual que relata Lahire (1995) en el libro mencionado, partir de un sujeto que actuase desde un vacío histórico sin pasado ni trayectoria, y al que segmentaríamos en la falacia de la relación entre usuario y orientador, que además a nuestro juicio se hubiera hecho incomprensible por este procedimiento reduccionista. Había que reconstruir a grandes rasgos, a través de las entrevistas en profundidad, los espacios y momentos esenciales en los que los sujetos van conformando sus visiones sobre el mundo educativo y laboral que les rodea, donde van tomando sus decisiones y donde se van conformando sus criterios y sus formas de ver el mundo del trabajo.

Se ha preguntado a los usuarios por su paso por el sistema educativo y por el papel jugado por la familia, a lo largo del proceso de conformación del objetivo profesional de las personas en búsqueda de empleo. Efectivamente, y como dice Lahire (1995: 18-20) resulta necesario y adecuado alejarse de los análisis mecanicistas, que tienden a dar por hechos una serie de presupuestos que en teoría obrarían de forma universal detrás de cada variable sociológicamente construida y que actúa como desencadenante principal de las hipótesis de trabajo. No toda familia donde el cabeza de familia posee un capital escolar y económico alto, implica necesariamente, una socialización en un hábitus propio de esa clase, ni se le puede suponer los mismos rigores en el enfoque familiar hacia la consecución de objetivos laborales a todas las familias de capital económico y escolar alto, por poner un ejemplo. 
Luego no es sólo la variable capital familiar la que conforma los hábitus, sino que serían los conjuntos de relaciones familiares los que actúan conformando los hábitus de los agentes, simplificando la explicación del autor mencionado.

Por lo tanto, lo que se ha intentado es descomponer variables como las formas y tipos de capital familiar, en relaciones que mantienen los usuarios con sus familiares en el ámbito de los procesos de formación de objetivos profesionales. Preguntas tan sencillas como las que enunciamos a continuación irían en la dirección descrita. ¿Qué relación tienen los usuarios con sus familiares antes y durante el desarrollo de los estudios? ¿Qué papel atribuye la persona que busca empleo a sus familiares en el momento en que tuvo que hacer elecciones en el sistema educativo? ¿Creen los chicos en busca de empleo que sus padres les entienden en cuanto a lo que están haciendo para formarse y para buscar empleo? ¿Supone una fuente de conflicto familiar el objetivo profesional que se marca la persona en busca de empleo? Respondiendo a esas preguntas, se reconstruyen las configuraciones familiares que conforman el hábitus pedagógico y laboral de los egresados universitarios.

\subsection{Hipótesis sobre estructuración de configuraciones laborales y su relación con la estrategia de construcción y persecución del logro educativo}

En primer lugar las relaciones que se dan entre la esfera de lo familiar y la formación de objetivos tanto formativos como laborales desmienten con toda claridad los presupuestos del enfoque del elector racional propios de las teorías económicas de la búsqueda de empleo, cuyos más destacados serían Becker y Stigler, fundamentalmente. Los individuos no eligen en el vacío de la asepsia económica, dónde estarían, según suponen estas teorías, haciendo cálculos sobre costes presentes y anticipando beneficios y costes futuros, a la hora de estudiar una carrera u otra, por ejemplo. Lejos de eso, la mayoría de las personas entrevistadas nos relatan como la elección de la carrera a estudiar se hizo en base al gusto personal por esos estudios, gusto que en muchos casos se declara (o se delata) claramente mediatizado por la influencia explícita e implícita de los padres, y que en otros casos (en estos la influencia es meramente implícita) no hace sino expresar, o actualizar un hábitus de clase en referencia al universo escolar que las personas van incorporando por su pertenencia y relaciones en la esfera familiar.

De esta forma, parecería que el sujeto, ni es libre en el sentido de tomar las decisiones desde su soledad personal (su personalidad psicológica), ni está obligado o forzado a tomar las decisiones de una forma imperativa (Bourdieu, 1991). Todo indica por lo visto en este trabajo que la decisión de estudiar una determinada carrera se toma influida por los valores y las constricciones inculcados, con mayor o menor grado de imposición externa por parte de la esfera familiar y el mundo de los pares en el que transcurre la vida cotidiana. Elegir una 
carrera o una formación siguiendo el principio del gusto, como hemos dicho, significa que estos actores valoran la formación, cuando se enrolan en ella como una actividad en sí misma (aprender, hacerse persona como nos dice una entrevistada que llamamos Eloisa) y no meramente como un fin para conseguir posición o renta futura como quieren las teorías del capital humano.

Si hay, como puede verse en algunos casos (como sería el de Elena, o también el caso de Ramón, por ejemplo), ciertos juicios y anticipaciones que las personas efectúan en el momento de hacer sus elecciones sobre la carrera a estudiar, y que a veces les llevan a distanciarse de lo que les marca su deseo a favor de otras opciones presupuestas como más rentables, no se trata tanto de la puesta en marcha de un cálculo racional en el sentido que lo querrían las teorías de Gary Becker, sino más bien de la actualización de un sentido práctico (Bourdieu, 1991: 94), en relación con el sistema educativo y laboral, que tiene su base, en todos los casos que hemos visto, en el seno de las configuraciones familiares de los buscadores de empleo.

\subsubsection{Dimensiones centrales sobre las que se articulan las configuraciones familiares}

Proponemos cuatro variables que se muestran centrales para definir las categorías de configuración familiar y comprender los distintos modelos con que pueden presentarse en el estudio de jóvenes buscadores de empleo, tal y como hemos planteado en este trabajo:

1. El capital escolar de los padres. Hay que considerar conjuntamente el capital del padre y la madre, que se traduce en una visión de educación y el acceso al empleo que es familiar.

2. El capital social de los padres. Se revela determinante en el momento de la carrera moral de acceso a los primeros empleos de calidad perseguidos.

3. El apoyo económico de los padres a sus hijos (solidaridad intergeneracional propia de un modelo cultural tradicional de familia). Se da por defecto, en todo tipo de configuraciones familiares, y por eso su relevancia es la menor para explicar las configuraciones familiares en términos generales.

4. El modelo comunicativo padres hijos, vinculado al estado de consolidación del estatus socio-laboral de los padres y madres. El modelo comunicativo parece instalarse con unos u otros condicionantes, no sólo en función del capital cultural y escolar de los padres, que es central en ello, sino en la trayectoria de clase en que se encuentra la familia.

En este trabajo se propone una tipología de configuraciones familiares, que mostramos a continuación. Resaltar que las configuraciones que nosotros pre- 
sentamos aquí no agotan el conjunto de las posibles, pero sí que resultan suficientes y exhaustivas para comprender sobre qué ejes se estructuran las interdependencias entre padres e hijos con respecto a la formación del logro ocupacional de estos últimos. En definitiva, nos muestran las claves con las que pueden estar forjadas las relaciones familiares en relación con la búsqueda de empleo.

\begin{tabular}{c|c|c|c|c}
\hline $\begin{array}{c}\text { Tipo config. } \\
\text { familiar }\end{array}$ & $\begin{array}{c}\text { Alto capital } \\
\text { escolar }\end{array}$ & $\begin{array}{c}\text { Exigencia } \\
\text { logro educativo } \\
\text { y laboral }\end{array}$ & $\begin{array}{c}\text { Logro } \\
\text { dialogado }\end{array}$ & $\begin{array}{c}\text { Capacidad } \\
\text { intervención } \\
\text { directa del capital social }\end{array}$ \\
\hline $\begin{array}{c}\text { Democrático } \\
\text { directivo }\end{array}$ & sí & sí/sí & sí o no & Puede darse o no darse... \\
\hline $\begin{array}{c}\text { Democrático } \\
\text { autónomo }\end{array}$ & sí & sí/sí & sí & Puede darse o no darse... \\
\hline $\begin{array}{c}\text { Impositivo } \\
\text { sin mandato } \\
\text { especifico }\end{array}$ & nó/no & no & no \\
\hline $\begin{array}{c}\text { Impositivo } \\
\text { con mandato }\end{array}$ & no & sí/sí & sí & no \\
\hline $\begin{array}{c}\text { Impositivo } \\
\text { con resultado } \\
\text { de abandono }\end{array}$ & no & no/no & no & \\
\hline
\end{tabular}

Algunas observaciones para entender el esquema de Configuraciones. Como el título académico se ha convertido en condición necesaria pero insuficiente para competir en el mercado laboral (Passeron, 1982), el capital económico juega en todas las configuraciones familiar, en función de su cantidad, por supuesto, por lo que es poco relevante para establecer las diferencias entre configuraciones. Al contrario, el capital cultural y escolar de los padres (padre y madre) es decisivo en la constitución del modo comunicacional distintivo de cada configuración. Y llegados al análisis de la carrera laboral, el capital social se relévela como determinante para conseguir los objetivos de logro laboral de los egresados universitarios.

El décalage intergeneracional que existe entre padres e hijos asociado al paso del fordismo a un modo de producción como podríamos llamar capitalismo financiero de consumo, afecta a todas las configuraciones familiares, pero se perciben los efectos más devastadores de las mismas en aquellas compuestas por los más bajos capitales culturales y sociales, especialmente. A estas configuraciones las hemos llamado Directivas, huyendo del empleo clásico de autoritarismo ba- 
sado en el modelo patriarcal, porque si bien corresponden a familias patriarcales, el rol del padre ha sido diluido con los avances de género ocurridos, por lo tanto sostenemos que no es posible el modelo patriarcal con personas que tienen o fabrican capital escolar, porque es rechazado de plano por los jóvenes. Lo que suscita problemáticas en carreras morales atrapadas en tales dilemas, que podrían representar los casos más probables de exclusión social, cuya base estaría en un sobre-cultivo del capital escolar frente a las necesidades del mercado y las propias expectativas y capacidad de comprensión de la familia.

\section{Configuraciones familiares, orientación laboral y trayectorias de búsquda de empleo}

Expondremos aquí con cierto nivel de detalle las carreras morales de los buscadores de empleo más representativos e ilustradores de la tipología de Configuraciones familiares que venimos de exponer. Dos de las trayectorias estudiadas, la de Elena y la de Aurelia , corresponden a un modelo de configuración familiar muy similar, que hemos llamado democrático directivo con la diferencia de que en el caso de Elena la familia muestra comprensión por los objetivos laborales de la hija, que no se ajustan plenamente a lo que ellos quieren, mientras que en el caso de Aurelia el progresivo deterioro de la relación entre la hija y sus padres, lleva al final a hacer del objetivo profesional y del logro de la chica un motivo de conflicto familiar. Serían por lo tanto dos variantes de una configuración familiar que comparte los siguientes rasgos: familias con alto capital escolar y situadas en la parte media-alta del estatus sociolaboral (funcionarios medios-altos, directores de banco, etc.). Son familias exigentes en términos de éxito profesional, sin capacidad aparente de intervención directa (con su capital social) en el ámbito en que sus hijos pretenden ubicarse, y familias enfocadas a que los hijos sigan preferentemente una carrera profesional propia del fordismo en el que ellos se instalaron. En este sentido como sucede con muchas familias, es la posición de funcionario la que se quiere para los hijos y a la que se incita.

Profundizando en el análisis del tipo de configuración democrático directivo, en la medida en que los padres dejan hacer a sus hijos dentro de unos marcos que pueden ir desde el diálogo, la comprensión y el acompañamiento continuo, señalando implícitamente (y a veces explícitamente) la obligación de no apartarse de la trayectoria laboral que los lleva al mantenimiento del estatus social consolidado por la familia. Aunque como hemos dicho el capital escolar es menos explicativo que el capital cultural y el capital social en el conjunto de las configuraciones familiares, los resultados del trabajo de campo mostrarían que el modelo Democrático Directivo sería quizás aquel en el que la estrategia de control y vigilancia continua de la carrera moral de los hijos, se basa más que ninguna otra en la inversión económica extraordinaria. 
La familia actúa con mecanismos de vigilancia continua a través de la propia negociación del objetivo laboral en este caso, y escolar en las fases precedentes de la carrera, donde los hijos gozan de una autonomía aparente al coste de no enfrentarse a la alta exigencia parental, en muchos casos no acompañada de capital social efectivo para la inserción en el campo laboral que se ha predeterminado. Ahí el estallido de conflictos dentro de la configuración familiar se hace presente, con diferentes grados de intensidad, obviamente.

El objetivo profesional del hijo llega en los casos vistos a convertirse en una forma de conflicto familiar a medida que evoluciona la carrera moral (Goffman, 1991) del buscador de empleo y los objetivos que se esperan de una y otra parte se van revelando como cada vez más distantes. Este es el caso de Aurelia en el momento en que se le hace la entrevista, donde la distancia progresiva entre la chica y la familia los padres estén incluso planteándose cambiar el orden de la configuración familiar, retirando el apoyo económico a la hija, apoyo que siempre recibió, junto con sus hermanos, con el objetivo de formarse y evitar un descenso de clase que es lo que los padres temen y quieren evitar. La amenaza no terminó por materializarse en los tres años de seguimiento, pero influyó en la relación con la familia. Aquí hay que destacar como un rasgo importante de estos modelos de configuración familiar, el hecho de que los padres aunque posean altos capitales formativos y económicos, no parecen poder movilizarlos de forma directa en el campo en el que sus hijos quieren formar sus objetivos profesionales.

En cuanto a la Configuración Familiar que llamamos de estilo Democrático Autónomo resulta muy similar a la descrita anteriormente en cuanto al tipo de capitales que las familias ostentan y a la estructura de estos capitales y su puesta a disposición y movilización en relación con el logro ocupacional de los hijos, y la connatural estrategia de mantenimiento de la posición de clase familiar para los descendientes. Lo que marcaría la diferencia sería fundamentalmente la instauración de un modelo comunicativo en el seno familiar, más orientado a hacer creer a los hijos que disponen de una amplia autonomía para elaborar y planificar sus carreras, dentro de un diálogo democrático donde se va consensuando en la familia, lo que les asegura a los padres que no se producen desviaciones de la norma de excelencia que van inculcando en los hijos a través del desarrollo de sus carreras morales. Se diría que comprometidos moralmente por la aparente flexibilidad y el apoyo incondicional de los padres a sus hijos (ninguno de los vistos se acaba saliendo del esquema de herencia de posición que se ha perfilado), les supone un compromiso moral mucho más elevado con respecto a sus padres, que en el modelo Directivo, lo que en los casos vistos, sin ausencia de ciertas fases de encontronazos, acaba traduciéndose en un éxito de la estrategia parental respecto a la carrera de los jóvenes. Esto a su vez implica, justamente, un aprovechamiento de la orientación laboral por parte de los chicos, de cara a producir justificaciones y auto-justificaciones sobre la validez personal en el seno de la configuración familiar, como explicamos con ejemplos más adelante. 
Es el caso de Ramón y Juana, que se insertan en Familias con padres que ostentan un capital social y escolar medio-alto, un estatus social alto consolidado, y que dan un aparente alto grado de maniobra y de autonomia a los hijos, aunque el grado implícito de exigencia por el éxito laboral y la conservación de la posición de clase familiar, lleva a que los chicos acaben aceptando las inevitables ventajas del proceso de herencia camuflado por la adquisición de títulos en el sistema educativo, (Bourdieu, los herederos?), que se materializa en la capacidad familiar de intervenir de forma directa con sus capitales en los ámbitos laborales de los hijos. Para decirlo con claridad, pueden enchufar a sus hijos en determinados puestos de trabajo.

Parecería, al menos en los casos vistos, que las posibilidades de herencia en lo laboral de que disfrutan estas personas en un mercado de trabajo muy mediatizado por los contactos para conseguir empleos de cierta calidad, no se producen a un coste cero para los propios beneficiarios. El caso de Juana muestra como, en un intento de alejarse de las posibilidades de inserción que le vienen heredadas, la chica intenta diversificar sus opciones, e incluso realiza algunos trabajos menos cualificados durante un tiempo, para darse a sí misma el sentido de una independencia que los favores familiares ineludibles le impedirían vivir con veracidad.

De hecho Juana parece mostrar su paso por algunas PAEs (paso con aprovechamiento) como pruebas que se pone a sí misma y que la auto-justifican en su capacidad de abrirse camino por sí misma, lejos del oficio que los familiares podrían ayudarle a heredar (su tío tiene una farmacia) pero que rechaza como objetivo profesional último. El caso de Ramón, que acepta de buen grado las posibilidades de herencia familiar en lo laboral, no deja de ilustrar también, el dolor y los encontronazos que suelen producirse en clases de altos capitales que se niegan en rotundo a que sus hijos se des-cualifiquen. La vigilancia continua de la trayectoria de Ramón en el sistema educativo que nos revela cuando reconoce que tuvo una época de gran conflictividad con sus padres debido a que era un mal estudiante, en los primeros años del bachillerato, da cuenta de estos costes que relatamos. Se da en el caso de Ramón una utilización (con aprovechamiento) de la orientación laboral que va en el mismo sentido que la explicada en Juana, en este caso si cabe de forma más llamativa aún. Si Ramón tiene toda una serie de facilidades y contactos que ya le han hecho disfrutar de trabajos en el mundo de la enseñanza derivados de procesos de herencia familiar, al mismo tiempo este hombre se esfuerza sobremanera en conseguir algún trabajo por sus propios medios, para lo cual activa todos sus capitales en un momento de su trayectoria (lo que le hace ser un modelo de persona empleable), y pasa por las políticas de empleo como una forma de demostrarse a sí mismo y a su entorno familiar, que si al final uno tiene que decantarse por la herencia, no es por incompetencia personal, sino porque los tiempos que corren así lo imponen. Ramón es válido, es capaz, pero tiene que heredar. Las políticas activas en estos casos parecen representar unos significados especiales para estas trayectorias. 
El caso de Cecilia ilustra perfectamente la pertenencia a una configuración familiar democrática autónoma, donde las posibilidades amplias de ayuda de la familia a la hija se corresponden a su vez, con la ubicación de ésta en un mercado laboral que ofrece oportunidades (por lo tanto casos excepcionales de inserción laboral temprana y con éxito en los mercados actuales) y además dentro de un clima familiar de perfecta armonía entre padres e hija, con respecto a las expectativas de estatus laboral y a los logros y objetivos profesionales marcados. Añadido al alto estatus familiar, en cuanto a capital económico, se da el fenómeno de que la familia posee capital social movilizable en el ámbito de inserción consensuado e incorporado por Cecilia.

¿Y qué uso hace esta chica de la Orientación Laboral? Cecilia utiliza los servicios de orientación como agencias de colocación, aparentemente también, como en los casos anteriores, para mostrar su capacidad de ubicarse laboralmente en el campo negociado con los padres y a la vez elegido y deseado por ella, pero en empresas distintas a las cercanas familiarmente. Y de hecho, es a través de los servicios de colocación de la bolsa de empleo de la Universidad como accede, con mucha rapidez, una serie de inserciones en su ámbito laboral, de poca entidad, pero que la lanzan ya a su carrera profesional. Aquí el único caso de uso de los servicios observado, del que se deriva una utilidad laboral específica de la orientación en la inserción laboral, aunque precaria e inicial.

Ya explicamos las incomprensiones entre padres e hijos a resultas de las irreversibles transformaciones de los mercados de trabajo actuales. Pero como era presumible, los impactos más amenazadores del consenso familiar, y de la convivencia en la institución, se encuentran mayoritariamente en los casos de familias de bajos capitales económicos y escolares, cuyo éxito en el sistema educativo no va seguido del esperado retorno en términos laborales y económicos para los hijos.

Con un mayor grado de conflicto familiar debido a la inversión a veces económica y otras, sobre todo afectiva que se ha realizado sería el caso de Eloisa. Y con menos grado de conflicto familiar y por lo tanto mayor apoyo moral dentro de la escasez de capitales que movilizar, sería el caso de Josefa. Los trazos comunes de ambos tipos de configuración familiar serian: padres desprovistos de capital escolar alguno y con escasos capitales económicos para sustentar los procesos de alargamiento de las trayectorias de acceso al empleo de sus hijos. Modelo de comunicación familiar sometido a las problemáticas de la incomprensión intergeneracional.

La presión moral sin mandatos específicos representa el tipo de configuración ilustrado por Eloisa. Las orientaciones muy especificas e inflexibles, constituirian el otro tipo de configuración que ilustra bien el caso de Josefa. Otros dos tipos de configuración familiar que hemos corroborado empíricamente en este trabajo. De Josefa hay que indicar que los padres la incitan a que siga estudiando oposiciones ahora, cuando en su momento se encontró sola a la hora de determi- 
nar cómo conseguir su logro laboral. Josefa llegó a trabajar como interina años atrás de su situación en el momento de la entrevista, en el que las vicisitudes de su carrera moral personal e individualizada la han desconectado de las enseñanzas medias, por lo que justamente utiliza la orientación laboral. Por eso accede a cursos de formación que puedan ir reconstruyendo una posición laboral de cierta analogía con la que tuvo y juzgó precaria en el pasado. Pero los cursos no la devolverán al punto en que abandonó la precariedad del interinato por movilidad laboral de su pareja. Aquí se evidencia, como en muchos casos, que los cursos de FPO no resultan completamente inútiles para los que los realizan, y fundamentalmente, como explicaremos con más detalle en el caso siguiente, le dan cierto sentido y esperanza a trayectorias laborales desmembradas por la sociedad del riesgo laboral. Pero no ubican socio laboralmente a sus usuarios.

Eloisa es hija de agricultor y ama de casa, con muy poco apoyo económico y con orfandad de la cultura legítima que se cultiva en la academia. La chica muestra la trayectoria educativa de quienes idolatran la cultura y el aprendizaje por el aprendizaje, quizás por su propia situación como persona perteneciente a una clase social popular subordinada. Habiendo estudiado filología inglesa, el primer gran encontronazo dentro de la configuración familiar se da cuando los padres esperan que ella opte a hacer oposiciones inmediatamente, mientras que la chica decide seguir formándose y trabajando precariamente al mismo tiempo. Seducida por el discurso legítimo de la formación continua en la sociedad del conocimiento (Castells, 2005), y tal vez presa de la inseguridad en sí misma que le da su doble condición de mujer en una sociedad patriarcal y de miembro/a de las clases populares, su situación de precariedad económica y laboral se va extendiendo en el tiempo, hasta que se convierte en un conflicto familiar abierto en el que pierde el apoyo moral del padre con quien ha dejado de hablarse, y apenas visita el domicilio familiar. Las posibilidades de caer en la exclusión social, tras haber forjado un capital cultural desde la soledad y la incomprensión familiar quedaron palpables en esta trayectoria, que muestra los peligros ocultos de la sociedad del riesgo de la que nos habla Ulrich Beck, para aquellas personas que parten a la competición individualizada sin un respaldo familiar suficiente, y presente en la mayoría de las trayectorias de egresados universitarios.

Y aquí los matices con los que hay que comprender las políticas activas de empleo que analizamos. Si bien se constata el carácter poco formativo de la relación de orientación laboral en sí misma, Eloisa representa un caso donde el paso a insertarse en cursos de Formación Profesional Ocupacional, concretamente como formadora de formadores, le insufla esperanzas en poder desembarcar en un universo de consolación laboral donde podría mantener una posición laboral digna frente a sus posibilidades de partida como persona carente de capitales. Por una parte la esperanza de trabajar como monitora de cursos, y por otra el insertarse en el ámbito de estos cursos que acogen a personas de similares trayectorias de clase, con sus vacilaciones y altibajos, ofrecen autoestima en tiempo 
real mientras se llevan a cabo, y aligeran el peso de la frustración de base que se debe al desfase entre esfuerzo y logro educativo y ausencia de éxito laboral y profesional.

Las PAEs, pueden ser útiles, pero en ámbitos de lo psicológico, lo personalfamiliar, como hemos visto, aunque nunca en el marco para el que fueron diseñadas: ser verdaderos mediadores del mercado laboral.

En el caso de Jesús vemos una configuración familiar del tipo Directivo con Mandato. Escasos capitales culturales y escolares de partida de padre y madre. El padre después de un proceso de reconversión empresarial pasó de empleado a montar un negocio de contable. Jesús tiene un tío que trabaja también como administrativo en el ámbito de la banca, lo que hace sugerir que en este caso, el escaso volumen de capitales escolares familiares habría que enmarcarlo, para su correcta comprensión sus trayectorias: diversos avatares indicarian que es una familia que se siente en proceso de ascenso social, lo que no deja de tener repercusiones sobre el tratamiento de la educación y los objetivos de los hijos en el ámbito familiar. Jesús está en el momento de la entrevista en condiciones de recibir una serie de impulsos y de guías desde el ámbito familiar para ingresar en el ámbito de la banca que resulta ser donde su familia tiene algún pequeño capital social que movilizar, y además un terreno laboral que confiesa que le gusta, sin duda después de haber estudiado la carrera de derecho impuesta-impulsada por los padres, en su estrategia de ascenso social. Por lo tanto Jesús, hace de la necesidad virtud, y adapta sus preferencias laborales en consonancia con los requerimientos familiares inculcados en su hábitus. Es tal vez este proceso de ascenso social familiar que no termina de consolidarse el que explique algo del discurso de Jesús, que aspirando a trabajar en el mundo de la banca y con pretensiones de conseguir algún puesto de relevancia con el tiempo, muestra al mismo tiempo, el discurso más cercano a los valores y la ética laboral de las clases populares, de cuantos hemos visto. Compelido a ascender, realizados los esfuerzos académicos que ello supone, pero confrontado al mercado laboral en sus primeros pasos, comprende intuitivamente la trampa de clase que supone el discurso meritocrático. Esto explicaría que tienda a identificarse ideológicamente como miembro de la clase trabajadora, en previsión de que el salto esperado no pueda consolidarse. Comprende a la perfección la solapada lucha de clases que se da en el acceso a los puestos relevantes en la sociedad, cuando se sale del escenario idealizado de la igualdad del marco académico.

La conclusión fundamental que podemos extraer de las relaciones entre modelos de configuración familiar y su relación con las políticas activas de empleo, es que la familia resta como la institución determinante tanto en la formación del objetivo educativo como en la del logro ocupacional, siendo estas políticas, en la práctica, meramente incidentales en las trayectorias de búsqueda de empleo, y en el acceso al mercado laboral de los jóvenes universitarios. Si autores como Pennel (2013:216) 
quieren, que el peso de los mediadores para el empleo como son los orientadores laborales, tanto públicos (Servicio Andaluz de Empleo), ONG, etc., como privados (ETTs), se acreciente inevitable y positivamente en los mercados post-flexibilizados, tales pretensiones pueden considerarse mucho más normativas que científicas.

Llegados al momento de buscar empleo tras haber finalizado su etapa universitaria, los jóvenes utilizan todos los mecanismos a su alcance para lograr primeras inserciones en su campo de formación o en los campos más afines. El apoyo económico familiar, y especialmente el capital social de la familia cobra un papel completamente decisivo dentro de la estrategia a seguir para conseguir el logro ocupacional, que en muchos casos, contrariamente a lo que podría creerse en una sociedad individualizada, viene consensuado y negociado hasta donde lo permite el décalage intergeneracional, con la familia. Los mediadores para el empleo, como los orientadores laborales, juegan un papel mínimo en relación a la orientación u reconversión de objetivos laborales, formulación de los mismos, y mucho menos en cuanto a una intervención directa ya sea creando capital social para los buscadores, ya sea propiciando esos primeros empleos, de los que se hayan, obviamente desprovistos.

Diseñadas en contextos económicos y sociales muy alejados tanto en el tiempo como en el espacio de los mercados laborales que rigen el presente en ciudades y regiones sin apenas tejido productivo, y basadas en trabajo en el sector servicios de poco valor añadido, una vez más, este análisis corrobora la escasa utilidad de tales políticas que funcionarían como instrumentos de inserción que crean alimentan un proceso de inserción circular e inacabable a partir de sí mismas (Mauger, 2002).

\section{Conclusión}

Planteadas a nivel nacional y europeo como la panacea para mejorar la empleabilidad y reducir las tasas de paro, las políticas activas de empleo sometidas al análisis empírico muestran su clara incapacidad de operar en el sentido que se les presupone. Su función tendría básicamente muy poco que ver con el mercado laboral y las inserciones efectivas, sino con acomodaciones de tipo psicológico y apaciguador del estrés con que los buscadores de empleo viven los mercados laborales de la globalización. Si hay algo que podrían hacer, en espacios regidos por un tejido productivo incapaz de generar empleos en el escenario de las nuevas tecnologías, sería sustituir la falta de capitales, sobre todo educativos y sociales de las familias de los jóvenes que buscan trabajo, tal y como hemos comprobado en universitarios.

Se demuestra que es de hecho, el capital social, constituido principalmente por la suma de capitales que acumula la configuración familiar a la que perte- 
nece el joven buscador de empleo, el que se revela decisivo en el acceso al trabajo, y en los procesos de transición de lo formativo a lo laboral. Capital no suficiente, pero casi completamente necesario para acceder a trabajos cualificados, bien remunerados, y propios del sector primario del mercado de trabajo de la ciudad donde se postulan los entrevistados.

En este sentido apuntamos aquí una tipología de configuraciones familiares en relación con el empleo, que si no exhaustiva ni definitiva, sí resulta operativa y operacionable en las ciencias sociales encargadas de dar cuenta de las transiciones educación-empleo, y de los procesos de acceso al empleo en nuestras sociedades contemporáneas. Queda patente en el trabajo que en escenarios laboralmente pauperizados, la familia es la institución que activa todos sus potenciales en sus luchas por impedir el desclasamiento de sus jóvenes, dejando al descubierto a ojos de los propios egresados universitarios la falta de correspondencia entre el discurso meritocrático que alientan la instituciones académicas y la realidad del mercado laboral.

\section{Bibliografía}

Alonso, L.E. (2000), Trabajo y postmodernidad: el empleo débil, Madrid, Fundamentos.

Beaud, S. y Weber, F. (2003), Guide de l'enquête de terrain, París, La Découverte.

BeCKer, G. (1994), Human capital: a theoretical and empirical analysis, with special reference to education, Chicago, Chicago University Press.

Bourdieu, P. (1991), La distinción: criterios y bases sociales del gusto, Madrid, Taurus.

- (2000), Poder, derecho y clases sociales, Bilbao, Desclée de Brouwer.

Castel, R. (1988): «Du travail social à la gestión sociale du non travail», en Revue Esprit, vols. 3-4, París, pp. 28-48.

- (1997), La metamorfosis de la cuestión social: una crónica del salariado, Buenos Aires, Paidós.

Castells, M. (2005), La era de la información: economía, sociedad y cultura. La Sociedad Red, Madrid, Alianza.

- (2005), La era de la información (vol. 1): economía, sociedad y cultura. La Sociedad Red. Ed. Alianza, Madrid.

Crespo Suárez, E. y Serrano Pascual, A. (2013), «Las paradojas de las políticas de empleo europeas: de la justicia a la terapia», en Universitas Psychologica, vol. 12, núm. 4: 1111-1124.

DARMON, I. (2006), "Formadores y usuarios frente al doble vínculo de la formación para la empleabilidad», en Cuadernos de Relaciones Laborales, vol. 24, núm. 2 : 37-62.

Erhel, C. (2010), «Les politiques de l'emploi en Europe: quelles réactions face á la crise?, en Centre d'Études de l'Émploi, n. ${ }^{\circ} 129$.

Goffman, E. (1970), Internados: ensayos sobre la situación social de los enfermos mentales, Buenos Aires, Amorrortu.

Lahire, B. (1995), Tableaux de familles. Heurs et malheurs scolaires en milieux populaires, París, Gallimard, Seuil. 
Martínez, J.S. (2007), "Clase social, género y desigualdad de oportunidades educativas», en Revista de Educación, núm. 342: pp. 287-306.

Mauger, G. (2002): «Las políticas de inserción. Una contribución paradójica a la desestabilización del mercado de trabajo", Cuadernos Andaluces de Bienestar Social, núm. 10, Granada, Escuela Universitaria de Trabajo Social, pp. 73-98.

Miguélez, Faustino y Prieto, Carlos (1999), Las relaciones de empleo en España, Madrid: Siglo XXI.

Passeron, J.C. (1982), «L'inflation des diplômes. Remarques sur l'usage de quelques concepts analogiques en sociologie ", Revue Française de Sociologie, volume 23, núm. 4 : 551-584.

Pennel, D. (2013), Travailler pour soi. Quel avenir pour le travail a l, heure de la revolution individualiste, París, Ed. Du Seuil.

Prieto, C. y Miguélez, F. (2009), «Transformaciones del empleo, flexibilidad y relaciones laborales en Europa». Política y Sociedad, vol. 46, núm. I y II: 275-287.

Sarasa, S. y Moreno, L. (comp.) (1995), El estado del bienestar en la Europa del Sur, Madrid, CSIC. 